ISSN: 2631-2654

https://doi.org/10.37135/unach.ns.001.01.06

Artículo de Investigación

\title{
Inclusión de pictogramas con la metodología aumentativa alternativa en el desarrollo de un tablero de comunicación para niños con Síndrome de Down \\ Inclusion of pictograms with the alternative augmentative methodology in the development of a communication board for children with Down Syndrome
}

\author{
Hernán Darío Centeno Aulla®i ${ }^{1}$, José Luis Bautista Zambrano², Juan Carlos Díaz \\ Ordóñez ${ }^{1}$, Gabriela Estefanía Román Santamaría ${ }^{3}$ \\ ${ }^{1}$ Dirección de Tecnologías de la Información y Comunicación, Escuela Superior Politécnica de \\ Chimborazo, Riobamba, Ecuador, EC060155 \\ ${ }^{2}$ Facultad de Informática y Electrónica, Escuela Superior Politécnica de Chimborazo, Riobamba, Ecuador, \\ EC060155 \\ ${ }^{3}$ Facultad de Recursos Naturales, Escuela Superior Politécnica de Chimborazo, Riobamba, Ecuador, \\ EC060155; jcdiazo@espoch.edu.ec \\ * Correspondencia: hernan.centeno@espoch.edu.ec
}

Recibido 14 mayo 2018; Aceptado 29 mayo 2018; Publicado 12 junio 2018

\begin{abstract}
Resumen: Dadas las carencias y obstáculos que se identifican en la comunidad para que las personas con Síndrome de Down (SD) alcancen el pleno reconocimiento de sus derechos, entre ellos a desarrollar una comunicación efectiva, en el presente trabajo se diseñó e implementó un tablero de comunicación web como una herramienta útil de información y expresión para niños con SD, basado en la metodología aumentativa alternativa para la identificación de pictogramas y emisión de mensajes. La arquitectura del sistema para la web es escalable, por lo tanto, acepta modificaciones y mejoras según la necesidad. Otra característica importante del tablero es la fácil usabilidad y accesibilidad. Para evaluar la funcionalidad se aplicó el método estadístico de Shapiro obteniendo como resultado un p-value igual a 0.2174 , es decir, que es mayor al 5\% de significancia, aceptando la hipótesis planteada. La solución presentada cumple las expectativas comunicacionales en cuanto a los elementos conceptuales y actitudinales, debido a que se hace uso del material utilizado en los salones de clase, los que están ajustados a las necesidades de aprendizaje de los niños, aportando a su desarrollo cognitivo y afectivo.
\end{abstract}

Palabras clave: Metodología aumentativa alternativa, Pictogramas, Síndrome de Down, Tablero de Comunicación.

Given the deficiencies and obstacles that are identified in the community so that people with Down Syndrome (SD) reach full recognition of their rights, including to develop effective communication, this work designed and implemented a web communication board as a useful information and expression tool among children with (SD), which is based on the alternative augmentative methodology for identifying pictograms and issuing messages. The architecture of the web system is scalable, therefore, it accepts modifications and improvements according to the

Abstract: need. Another important feature of the board is the easy usability and accessibility. To evaluate the functionality, the statistical method of Shapiro was applied, obtaining as a result a p-value equal to 0.2174 that is greater than 5\% of significance accepting the proposed hypothesis. The presented solution fulfills the communicational expectations regarding the conceptual and attitudinal elements, due to the use of the material used in classrooms, which are adjusted to the learning needs of children, contributing to their cognitive and affective development.

Keywords: $\quad$ Alternative augmentative methodology, Pictograms, Down Syndrome, Communication Board. 


\section{Unach}

\section{$1 \quad$ Introducción}

Los niños con SD, para su educación y comunicación, hacen uso de pictogramas que son imágenes en blanco y negro, las mismas que se encuentran impresas en tarjetas pequeñas almacenadas en libros voluminosos por la cantidad de imágenes que se compone, estos pictogramas se encuentran clasificados de acuerdo al mensaje que expresan, los niños Down deben comprender qué expresa cada pictograma para poder formar oraciones y emitir un mensaje, por la condición que se lleva estos pictogramas y al ser imágenes a blanco y negro, el niño Down pierde el interés de hacer uso de estos tableros físicos, por lo que no logran concentrarse en las actividades que están realizando.

A raíz de esta problemática presentada se optó por desarrollar un tablero virtual de comunicación para la web, el cual sirva de apoyo en la comunicación y formación de niños Down incluyendo pictogramas de colores que llamen la atención de los usuarios. Para evaluar el funcionamiento del tablero se plateó la siguiente hipótesis nula $(H O)$ y una alterna $(H 1)$ :

H0: La utilización del tablero web mejorará la comunicación de un niño con SD.

H1: La utilización del tablero web no mejorará la comunicación de un niño con SD.

La existencia del SD o "mongolismo", término con el que históricamente se le denominó, se remonta desde el siglo VII d.C., considerada como una enfermedad genética resultante de la trisomía del par 21 por la no disyunción meiótica, mitótica, que se presenta con una frecuencia de 1 en 800.000 habitantes, incrementándose con la edad materna. Desde esta perspectiva, las personas con SD presentan signos clínicos generales como: deficiencia mental, braquicefalia, eritema facial continuo, microtia, manchas de Brushfield, anomalías cardíacas congénitas, displasia de la segunda falange del quinto dedo, manos pequeñas, facies mongólica, hipotonía, además de retraso en el desarrollo físico y psíquico (Pérez, 2014) algunas de sus características físicas los hace semejantes a los nativos mongoles de Asia (Gómez y Cruz, 2008). Dichas manifestaciones tendrán relación con aspectos fundamentales en el lenguaje, afectando tanto la adquisición como el desarrollo del mismo, ya sea en la expresión oral como en la comprensión. Por lo tanto, dicho déficit lingüístico afectará las habilidades comunicativas y estas a su vez afectarán al desarrollo individual e integración social de las personas si no se refuerza continuamente su comunicación (Cortes y Romero, 2015). En forma adicional, se debe manifestar que no existe tratamiento para curar el SD, por lo que las medidas de rehabilitación que se realizan son mediante técnicas especiales tales como fisioterapéuticas, fonoaudiológicas, psicológicas, que juegan un papel importante permitiéndoles en la mayoría de las veces una adecuada reinserción social (Pérez, 2014). Sin embargo, la presencia de retraso mental en estos pacientes puede ser variable, encontrándose afecciones leves que permiten a estos pacientes realizar labores cotidianas con mucha facilidad (Pérez, 2014).

El cerebro no es una caja cerrada y terminada, sino un proceso en constante cambio y evolución para responder a los estímulos que recibe del exterior y la forma más natural de llegar al mismo es a través de sus interacciones con el medio que le rodea (Villa y Martínez, 2008). Con ello, la comunicación surge de lo más profundo de la naturaleza humana, en su dimensión personal y social; de hecho, es el resultado del ejercicio de la naturaleza social de la persona que le permite representar y manejar la realidad en forma esencialmente compartida (Salazar, Ferrer y Toro, 2017) a través del lenguaje que es una facultad semiótica, la cual permite simbolizar la realidad, así como desarrollar el pensamiento y entablar relaciones a través de cualquier medio; mediante distintos signos y códigos que se producen en el contexto de una cultura (Vergara, 2008). Por consiguiente, la capacidad de efectuar actos de comunicación comprensibles es lo que permite al ser humano interactuar como sujetos activos en un entorno social (Sotillo, 1993) si faltara la comunicación predominaría la dependencia y el aislamiento. A su vez llamamos comunicación alternativa a cualquier forma de comunicación distinta del habla y empleada por una persona en contextos de comunicación cara a cara, a través del uso de signos manuales y gráficos, el sistema morse, la escritura u otros.; por otra parte, la comunicación aumentativa significa comunicación de apoyo o de ayuda (Tetzchner y Martinsen, 1993). En este contexto nace la metodología de comunicación aumentativa alternativa (CAA) que se orienta al desarrollo de competencias comunicacionales de personas con discapacidad en el lenguaje (Guisen y Sanz, 2014) basada en un conjunto definido de códigos no vocales (lingüísticos y no lingüísticos) necesitado o no de soporte físico, que permiten representar conceptos y llevar a cabo actos de comunicación; que sustituyen o complementan a las tradicionales prácticas de expresión (habla y escritura), cuando por sí solas no son suficientes para entablar una comunicación efectiva con el entorno (Abril, Delgado y Vigara, 2012; Basil, Soro y Rosell, 1998). No obstante, se consideran una ayuda tecnológica que habilita a los usuarios de CAA a la interacción y toma de decisiones personales en el contexto social que los rodea, y propicia las condiciones para su integración al sistema educativo e inclusión social (Dorda, González y Adrián, 2004; Hurtado y Soto, 2005).

El gran desarrollo tecnológico que se ha producido recientemente ha propiciado lo que algunos autores denominan la nueva "revolución" social, con el desarrollo de la sociedad de la información (Belloch, 2012) en este sentido, la presencia de las TIC en 


\section{Unach}

cualquier actividad humana es imparable, su utilización está provocando un cambio hacia la mejora del conjunto de la sociedad y en consecuencia de la calidad de vida de los ciudadanos (Domínguez, 2003), las TIC son motivadoras e incrementan la autoestima (Roqueta y Hernández, 2005), atendiendo únicamente a su influencia sobre las variables psicológicas del aprendiz que opera con un ordenador y que se relaciona (Coll, 2008) a través de él por el hecho de vivir en un mundo de evolución tecnológica constante, la cual está diseñada para que su uso sea más fácil e intuitivo al presentar interfaces llamativas en los diferentes dispositivos existente, que con solo una imagen describen el proceso de una determinada aplicación (Valero, Redondo y Palacín, 2012). En particular, los sistemas de CAA asistidos de alta tecnología (tecnología informática) incluyen software de CAA. Si bien el usuario de estos sistemas no abandona el empleo de instrumentos de baja (tablero o carpeta de comunicación) y media (comunicador electrónico) tecnología, su incorporación, ya sea de manera temporaria, permanente o sólo como un instrumento ligado al espacio educativo y/o terapéutico, se concibe una tendencia institucionalizada debido a las ventajas que la tecnología informática provee para el alcance de la comunicación autónoma en comparación con los anteriores (Montoya, 2002). Pocos avances tecnológicos han tenido un impacto similar a la web en la historia de la humanidad, pues en menos de una década se ha convertido en un medio de comunicación prácticamente indispensable y en la principal fuente de información para parte importante de la población a nivel mundial (Baeza, Rivera y Velasco, 2004).

Bajo esta perspectiva, la presente investigación tuvo como objetivo de estudio la inclusión de pictogramas con la metodología aumentativa alternativa en el desarrollo de un tablero de comunicación para niños con Síndrome de Down, diseñado con el fin de optimizar el proceso de comunicación, propiciando accesibilidad y usabilidad a los usuarios beneficiaros, potenciando de tal manera al usuario a tener un acto comunicativo. Dentro de los objetivos específicos de estudio fueron: identificar los pictogramas y el mensaje que transmite cada uno, definir la arquitectura del sistema para la web, desarrollo del sistema para la web y evaluar la funcionalidad del sistema mediante un método estadístico. Con el propósito principal de contribuir al desarrollo cognitivo y social, mejorar el proceso de integración en la familia, la escuela, la sociedad y el trabajo (Salazar, Ferrer y Toro, 2017).

\section{Metodología}

El desarrollo del tablero para la web para niños con SD estuvo basado en dos metodologías: la primera que hace referencia a la fundamentación del sistema y la segunda que está relacionado con la definición y desarrollo del sistema web.

\subsection{Metodología aumentativa alternativa}

La metodología aumentativa alternativa se refiere al conjunto de estrategias y métodos más adecuados para complementar la comunicación oral o escrita. Según von Tetzchner (1993), un sistema de comunicación aumentativa y alternativa puede funcionar como lenguaje de apoyo en personas que presentan retraso en el desarrollo del lenguaje, como es el caso de niños con SD.

De manera específica se presenta la identificación de pictogramas y el mensaje que transmiten los niños con SD.

Los pictogramas que son usados en las aulas escolares se encuentran impresos en pequeñas tarjetas, las que están compuestas por imágenes en blanco y negro que transmiten un determinado mensaje. Cada pictograma está clasificado de acuerdo a las actividades que realizan los niños con $\mathrm{SD}$, esta clasificación se divide en: pronombres, sociales, adjetivos, verbos, sustantivos, alimentos, bebidas, frutas, golosinas, granos, ropa, juguetes, tiempo, días, módulo de meses, colores, números y letras. Este proceso de clasificación fue parte de la fundamentación del sistema web.

\subsection{Definición de la arquitectura del sistema para la web}

La arquitectura de información se ocupa del diseño estructural de los sistemas de información, su problema central es la organización, recuperación y presentación de información mediante el diseño de ambientes intuitivos (Baeza, Rivera y Velasco, 2004) siendo el objetivo final de la arquitectura de información el organizar la información de forma coherente. Ello proporcionará a los usuarios un acceso rápido y fácil a la información necesaria, pues de una arquitectura correcta depende, en gran medida, el buen funcionamiento de un sitio y su utilidad (Arabito, 2003; Pérez, 2003).

En este sentido, para el desarrollo de todo sistema informático en primera instancia se definió la arquitectura a seguir para su correcto desarrollo, con la integración de pictogramas y del speech de la web se construyeron los tableros de comunicación virtuales. Para mejorar la estructura del sistema se aplicó la arquitectura de tipo modelo vista controlador en donde el usuario final selecciona un pictograma mediante la vista, y desde ahí realiza la petición al controlador, el controlador a su vez realiza una petición al modelo, quien devuelve la respuesta al controlador y esta a su vez muestra la respuesta por medio de la vista al usuario final, el proceso se ilustra en la figura 2. 


\section{Unach}

\subsection{Desarrollo del sistema para la web}

Para el desarrollo de los tableros virtuales de comunicación se tomó como punto de partida la metodología de enseñanza utilizada en niños con SD. Se realizó el análisis de las herramientas empleadas en el proceso de formación y comunicación, por esta razón se realizó un trabajo coordinado periódico en el que participaron los profesores y alumnos de los institutos de educación especial involucrados en el proceso investigativo. En primera instancia, mediante observación directa, se analizó el proceso de enseñanza-aprendizaje dentro de las aulas, luego de diferentes terapias de lenguaje y comunicación, se identificó que los niños en sus terapias y aulas de clases manejan imágenes en blanco y negro (pictogramas) con el fin de comunicarse. Las imágenes transmiten un mensaje, cumpliendo el papel de intermediario entre el niño con SD y el receptor. Los pictogramas se encuentran clasificados en función a las actividades o mensajes que transmiten los mismos, que se llevan en libros en forma de tarjetas, el niño con SD a medida que pasa por un proceso de educación aprende a usar, diferenciar y clasificar adecuadamente los pictogramas. Como resultado, aprenden a usarlos como medio de comunicación que transmite un mensaje. Cuando el niño quiere emitir un mensaje debe buscar cada tarjeta del libro de comunicación e ir formando oraciones sobre una mesa que consiste de un tablero de comunicación. En los tableros de comunicación el niño con SD aprende a reconocer el mensaje que transmite cada pictograma, de tal forma ellos pueden relacionar su entorno con los pictogramas, además permite formar frases y pueden realizar varias otras tareas. Todo el proceso descrito es de forma manual, por lo cual al poco tiempo de hacer uso de los tableros físicos de comunicación los niños se distraen con facilidad, debido a que se notó la pérdida de interés por su uso, partiendo de esta necesidad y con el objetivo de evitar que los niños, maestro y padres de familia tengan que llevar un tablero físico se automatizó el proceso por medio de tableros web, cuya finalidad fue la de lograr una mayor interacción comunicacional, a partir de una interfaz llamativa.

Varias herramientas fueron aplicadas para el desarrollo del sitio web, seleccionando aquellas que son gratuitas, evitando así la necesidad de adquirir licencias. Entre ellas:

Lenguaje de marca HTML5: Conjunga el estilo simplificado y práctico con el rigor técnico necesario para que el lector saque el mayor partido. HTML ha evolucionado a la especificación HTML5, que es una visión madura y eficiente para unificar criterios a la hora de programar la interfaz del usuario de un documento web (Oros, 2008).
Hojas de estilo CSS: permiten a los usuarios especificar su propia hoja de estilo local que será empleada en una aplicación web, con lo que aumenta considerablemente la accesibilidad, por ejemplo, personas con deficiencias visuales pueden configurar su propia hoja de estilo para aumentar el tamaño del texto o remarcar más los enlaces (Hernández y Greguas, 2010).

Java Script: no es más que un sencillo lenguaje de programación que presenta una característica especial: sus programas, llamados comúnmente scripts, se abren en las páginas HTML y se ejecutan en el navegador (Netscape Navigator y Microsft Explorer). Estos scripts normalmente consisten en unas funciones que son llamadas desde el propio HTML cuando algún evento sucede. De ese modo, podemos añadir efectos como que un botón cambie de forma al pasar el ratón por encima, o abrir una ventana nueva al pulsar en un enlace (Navarrete, 2006)

Motor de base de datos MySql: permite la construcción, de una manera sencilla y eficiente, de verdaderos sitios web dinámicos. En los últimos tiempos se está observando cómo son cada vez más las empresas que optan por estas tecnologías para el desarrollo de sus portales; incluso, en el caso de particulares, algunos de los servicios gratuitos de alojamiento de páginas web ofrecen la posibilidad de usar PHP en conjunción con MySQL (Cobo, Gómez, Pérez y Rocha, 2005).

PHP: es un lenguaje de programación interpretado, diseñado originalmente para la creación de páginas web dinámicas. A partir de PHP 5 incorpora un modelo de objetos más completo. Entre sus características están la inclusión de la visibilidad, las clases abstractas y clases y métodos finales, manejo de excepciones, interfaces, clonación y tipos sugeridos (Acosta, Greiner, Dapozo y Estayno, 2012).

Finalmente, para evaluar la funcionalidad del tablero virtual se aplicó como método la encuesta y como instrumento el cuestionario que fueron usados para obtener información la misma que se encuentra estructurada con 10 preguntas de tipo cerradas, dirigida a 33 padres de familia.

\section{Resultados y Discusión}

La capacidad de efectuar actos de comunicación comprensibles es lo que nos permite interactuar como sujetos activos en nuestro entorno social utilizando la comunicación, como práctica social, es condición para la autonomía; sin ella predomina la dependencia y el aislamiento (Guisen, 2014). Con ello, los Sistemas de CAA son instrumentos de 


\section{Unach}

enseñanza-aprendizaje (Sotillo, 1993). Con esa visión, debido a la dificultad que presentan los niños con SD para efectuar el acto de comunicación, su proceso de formación y aprendizaje está basado a través de pictogramas, siendo imágenes en blanco y negro las cuales transmiten un determinado mensaje, se encuentran impresos en tarjetas que sirven para la formación de tableros de comunicación, al mismo tiempo son plasmados en libros voluminosos por la existencia de una gran cantidad de pictogramas que se utilizan. El uso de los tableros físicos de comunicación para el niño con $\mathrm{SD}$ en su edad temprana es llamativo y atractivo, pero con el paso del tiempo los niños pierden el interés. La dificultad se encuentra en que los niños deben llevar todos estos pictogramas impresos, lo que hace que su portabilidad sea dificultosa, trayendo como consecuencia la distracción y el poco interés de su uso. Con esta necesidad de mejorar la interacción de comunicación con los niños con SD con los pictogramas se procedió al desarrollo de tableros virtuales de comunicación que se adaptan a cualquier tipo de dispositivo a nivel tecnológico, para su diseño tuvieron como base los pictogramas y la metodología de aprendizaje y comunicación usado por docentes especialistas en enseñanza Down.

Como manifiestan Heredero y Carralero (2014), las innovaciones tecnológicas, que se están produciendo en la sociedad con el objetivo de facilitarnos la vida, han hecho que se replantee la forma de enseñar. Una de las aplicaciones gratuitas diseñadas por las maestras de educación especial, Laura Muñino Gil y Guadalupe Montero, en el año 2011, dio vida a LUA, el personaje que guía la lectura global (Heredero y Carralero, 2014). Aplicación que ha sido utilizada en dispositivos táctiles como es el caso de pizarras digitales y tablets, dirigida a personas con dificultades en lectoescritura, con incapacidad de comunicarse, con y sin necesidades educativas especiales.

\subsection{Identificación de pictogramas y el mensaje que transmite}

Se identificaron pictogramas que utilizan los niños con SD en su proceso de comunicación, tomados de un libro físico, los cuales fueron escaneados, procesados en un formato adecuado y alojados en una base de datos figura 1 .

Las imágenes se pueden considerar sencillas en comparación con los signos manuales en la enseñanza de niños muy pequeños por varias razones: en primer lugar, las imágenes se asemejan más a sus referentes que los signos manuales, haciéndolos fáciles de entender; en segundo lugar, apuntando hacia o llegar a una imagen para hacer una solicitud implica menos esfuerzo físico y planificación motora que ejecutando un signo manual; y tercero, las imágenes son carga de memoria de recuperación reductora estacionaria (Mirenda, 2003).

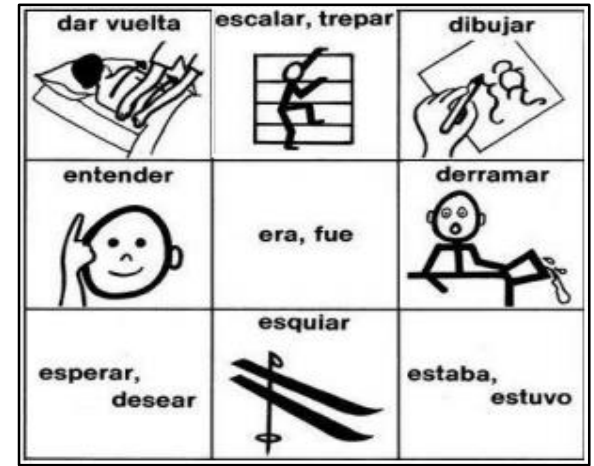

Figura 1: Pictogramas del Tablero de Comunicación

\subsection{Definición de la arquitectura del sistema para la web.}

En cuanto a la definición de la arquitectura del sistema para la web, se contempla la organización de la información desde una perspectiva amplia. Incluye tanto a la estructura propuesta para el contenido como a los mecanismos necesarios para conocer y explorar dicha estructura en su presentación visual (Pérez, 2003).

En la arquitectura del sistema se puede definir como está estructurado. El tablero para la web está integrado por un motor de base de datos en donde se alojan los pictogramas con su significado, la base de datos alimenta al tablero virtual que es utilizado por los niños con SD. Cabe mencionar que los sistemas CAA basados en el uso de recursos de alta tecnología, aprovechan la pantalla dinámica o tablero como forma de funcionamiento y le suman salida de voz. Estos sistemas ofrecen gran flexibilidad y resultan aplicables a la posible diversidad de niveles cognitivos y funciones de comunicación que pueden presentar las personas con trastornos severos y/o moderados del habla (Cárdenas, Gil, Gómez y Ríos, 2001).

\subsection{Desarrollo del sistema para la web.}

De esta manera se desarrolló un sistema web, el mismo que está compuesto por 18 módulos que corresponden a la clasificación dada por los expertos en procesos de enseñanza. Se agruparon en 18 grupos principales en función a las actividades que transmiten cada uno de los pictogramas que se encuentran dentro de las siguientes denominaciones: adjetivos, alimentos, bebidas, colores, días, frutas, golosinas, granos, juguetes, letras, módulo de meses, números pronombres, ropa, sociales, sustantivos, tiempo y verbos. La vista del tablero de comunicación para la web se presenta en la figura 3. 


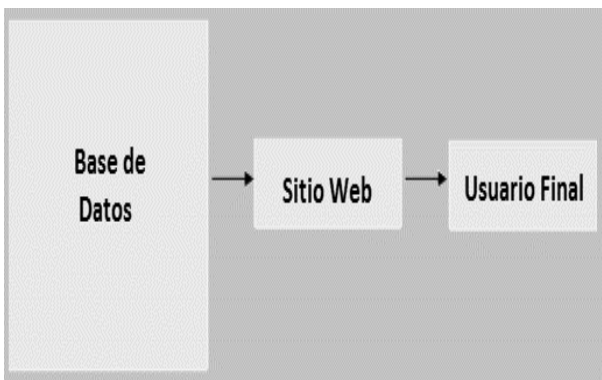

Figura 2: Arquitectura del Sistema para la Web

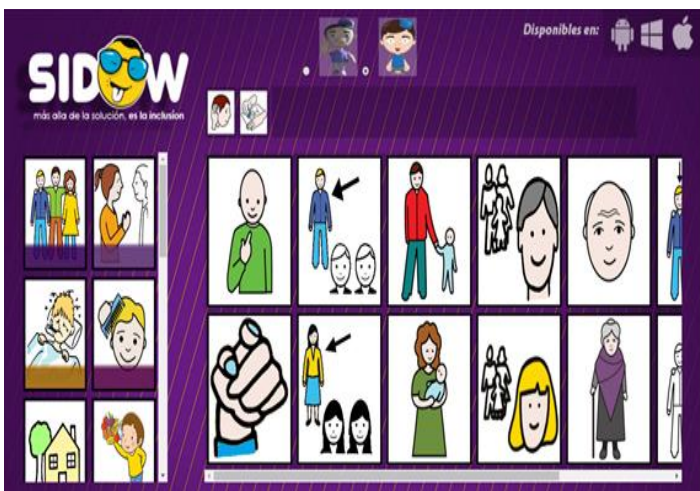

Figura 3: Captura de Pantalla del Tablero de Comunicación para la Web

\subsection{Evaluación de la funcionalidad del sistema mediante un método estadístico}

Con el fin de aceptar o rechazar la hipótesis nula se planteó una encuesta a los padres de los niños Down de donde se obtuvieron los siguientes datos mostrados en la tabla 1.

El análisis de los datos obtenidos se hizo por medio de Shapiro test, como se indica en la figura 4, de donde se obtiene que p-value igual a 0.2174, es decir, que es mayor al $5 \%$, lo que significa que la hipótesis nula se acepta.

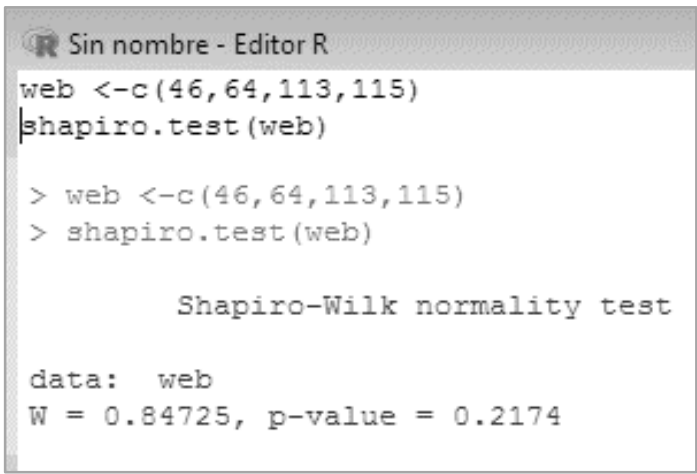

Figura 4: Análisis de datos

Tabla 1: Datos obtenidos de las encuestas realizadas.

\begin{tabular}{|c|c|c|c|c|c|c|}
\hline No. & Criterios de evaluación & Bajo & Medio bajo & Medio & $\begin{array}{l}\text { Medio } \\
\text { Alto }\end{array}$ & Alto \\
\hline 1 & $\begin{array}{l}\text { Califique del desempeño de su hijo con la } \\
\text { aplicación }\end{array}$ & 0 & 3 & 7 & 10 & 13 \\
\hline 2 & $\begin{array}{l}\text { Califique los gráficos de la aplicación de acuerdo } \\
\text { como interactúan su hijo. }\end{array}$ & 0 & 5 & 8 & 3 & 17 \\
\hline 3 & Califique la emoción de su hijo con la aplicación. & 0 & 4 & 7 & 18 & 4 \\
\hline 4 & $\begin{array}{l}\text { Califique como interactúa el niño con las } \\
\text { actividades cotidianas asociando con la aplicación }\end{array}$ & 0 & 2 & 6 & 10 & 15 \\
\hline 5 & $\begin{array}{l}\text { Califique la aplicación en la ayuda del desarrollo } \\
\text { de aprendizaje en el niño }\end{array}$ & 0 & 3 & 12 & 10 & 8 \\
\hline 6 & $\begin{array}{l}\text { Califique la aplicación en la ayuda del desarrollo } \\
\text { de aprendizaje en el niño }\end{array}$ & 0 & 2 & 10 & 15 & 6 \\
\hline 7 & $\begin{array}{l}\text { Califique la facilidad de acceso a la cada } \\
\text { aplicación. }\end{array}$ & 0 & 5 & 6 & 12 & 10 \\
\hline 8 & $\begin{array}{l}\text { Califique el despliegue (Facilidad de acceso a la } \\
\text { aplicación a través de diferentes dispositivos } \\
\text { móviles o de escritorio). }\end{array}$ & 0 & 9 & 4 & 16 & 4 \\
\hline 9 & $\begin{array}{l}\text { Como califica el cambio de aprendizaje de su hijo } \\
\text { con el sitio web }\end{array}$ & 0 & 9 & 1 & 9 & 14 \\
\hline \multirow[t]{2}{*}{10} & Como califica el uso del sitio de su hijo. & 0 & 4 & 3 & 10 & 16 \\
\hline & Total & 0 & 46 & 64 & 113 & 115 \\
\hline
\end{tabular}




\section{Unach}

\section{Conclusiones}

Se presentan las siguientes conclusiones en función a los objetivos específicos planteados:

Se logró obtener un libro físico, mismo que contiene los pictogramas que son utilizados por los niños con SD con el objetivo principal de incluir en el sistema web, a través de escanear el libro y a su vez el recortar las imágenes y guardar cada pictograma en un formato adecuado para alojar en una base de datos clasificándolos de acuerdo a su mensaje que transmite al momento de realizar la comunicación. Además, se estableció una arquitectura fácil de ser implementada que es de gran ayuda al desarrollo del sitio, la arquitectura definida divide al sistema en 18 módulos, mismo que si se requiere hacer algún cambio solo se modificará el módulo especificado sin alterar al resto del sistema, porque fue diseñada pensando en que un sistema informático está sujetos a cambios y mejoras.

Finalmente, como resultado final de la integración entre los pictogramas identificados y la arquitectura del sistema se generó un tablero de comunicación virtual para la web para ayudar a los niños con síndrome de Down a lograr una comunicación interactiva e incluyente; el tablero a más de que permite visualizar los pictogramas, también dicen el significado de cada uno a través del speech del web, para lo cual se utilizó JavaScript.

\section{Referencias}

Abril, D., Delgado, C. \& Vigara, A. (2012). Comunicación aumentativa y alternativa. Guía de referencia. Madrid: CEAPAT.

Acosta, J., Greiner, C., Dapozo, G., \& Estayno, M. (2012). Medición de atributos $\mathrm{POO}$ en frameworks de desarrollo PHP. XVIII Congreso Argentino de Ciencias de la Computación.

Arabito, J. (2003). Observatorio de la sociedad de la información: De la catedral al libro y del libro al Bazar de las Redes. Las nuevas tecnologías de la información y la comunicación en la transformación social y cultural de principio del milenio. Recuperado de http://www.tierralibredigital.com.ar./obv_soc_info_n ota13.htm

Baeza, R., Rivera, C. \& Velasco, J. (2004). Arquitectura de la información y usabilidad en la web. El profesional de la información, 13(3), 168-178.
Como trabajo a futuro se piensa implementar tableros de comunicación para dispositivos móviles que sean intuitivitos y estén disponibles sin necesidad de tener conexión a internet, además se recomienda realizar un estudio más amplio en la comunicación de personas Down con el objetivo de descubrir nuevas técnicas y herramientas que sirvan de apoyo para llevar una buena comunicación.

\section{Conflicto de Intereses}

Se expresa de manera libre y voluntaria que en el desarrollo del presente artículo no existen conflictos de interés personales por parte de ninguno de los autores por lo que se autoriza la publicación del trabajo.

\section{Agradecimientos}

Los investigadores agradecen de manera encarecida a los niños con Síndrome de Down que participaron en el proyecto, a sus padres y a sus maestros por el apoyo brindado durante el desarrollo del sistema que resultó ser de ayuda en su comunicación.

Basil, C., Soro, E. \& Rosell, C. (1998). Sistemas de signos y ayudas técnicas para la comunicación aumentativa y la escritura: principios teóricos y aplicaciones. Barcelona: Masson.

Belloch, C. (2012). Las Tecnologías de la Información y Comunicación en el aprendizaje. Material docente (on-line). Departamento de Métodos de Investigación y Diagnóstico en Educación. Universidad de Valencia. Recuperado de http://www.uv.es/bellochc/pedagogia/EVA1.pdf

Cárdenas, M., Gil, A., Gómez, V. \& Ríos, A. (2001). El uso de pantalla dinámica en comunicación aumentativa-alternativa. $V$ Jornadas de SIDARIBERDISCAP

Cobo, A., Gómez, P., Pérez, D. \& Rocha, R. (2005). PHP y MySQL, Tecnologías para el desarrollo de aplicaciones web. Madrid: Ediciones Díaz de Santos.

Coll, C. (2008). Psicología de la educación virtual: aprender y enseñar con las tecnologías de la información y la comunicación. Madrid: Ediciones Morata. 


\section{Unach}

Cortes, C. \& Romero, F. (2015). Síndrome de Down (Tesis de Grado inédita). Universidad de Aconcagua, Sede Quilpué.

Domínguez, M. (2003). Las tecnologías de la información y la comunicación: sus opciones, sus limitaciones y sus efectos en la enseñanza. Nomadas revista Critica de Ciencias Sociales y Juridicas, 8, 1-68.

Dorda, J., González, J. \& Adrián, M. (2004). De las ayudas técnicas a la tecnología asistida, en Tecnología, Educación y Diversidad: Retos y Realidades de la Inclusión Digital. Actas del III Congreso Nacional de Tecnología, Educación y Diversidad (Tecnoneet).

Gómez, J. \& Cruz, S. (2008). Síndrome de Down. Carta de la Salud, 148, 1-2. Recuperado de www.valledellili.org/media/pdf/cartasalud/CSSeptiembre_2008.pdf.

Guisen, A. (2014). Colaboración y comunicación aumentativa mediada por TIC. XVI Workshop de Investigadores en Ciencias de la Computación.

Guisen, M. \& Sanz, C. (2014). Diseño de ECCA (Entorno Colaborativo de Comunicación Aumentativa y Alternativa). Una ayuda tecnológica para alumnos con Necesidades Complejas de Comunicación. Edutec-e, Revista Electrónica de Tecnología Educativa, 50, 1-16.

Heredero, E. \& Carralero, A. (2014). Experiencias y recursos con las tics para la atención al alumnado con necesidades educativas especiales. Acta Scientiarum, 36(2), 279-286.

Hernández, R. \& Greguas, D. (2010). Estándares de Diseño Web. Ciencias de la información, 41(2), 6971.

Hurtado, M. \& Soto, F. (2005). Tecnologías de ayuda en contextos escolares. Murcia: Consejería de Educación y Cultura.

Mirenda, P. (2003). Toward functional augmentative and alternative communication for students with autism: Manual signs, graphic symbols, and voice output communication aids. Language, Speech and Hearing Services in Schools, 34, 203-216.

Navarrete, T. (2006). El lenguaje JavaScript. Curso Fonaments y Cartografia i SIG. Departamento de Tecnologías de la Información y las Comunicaciones, Universitat Pompeu Fabra.

Oros, J. (2008). Diseño de páginas web con Xhtml, Javascript y Css, 3era edición. Madrid: Editorial RA-MA.

Pérez, D. (2014). Síndrome de Down. Revista de Actualización Clínica Investiga, 45, 2357:2361.

Pérez, J. (2003). Diseño informacional de los sitios web. Acimed. 11(6).

Roqueta, C. \& Hernández, B. (2005). Recursos para la aplicación de las nuevas tecnologías en la educación del alumnado con necesidades educativas derivadas de discapacidad visual en edades tempranas. Integración: Revista Sobre Ceguera y Deficiencia Visual, 46, 13-22.

Salazar, N., Ferrer, Y. \& Toro, I. (2017). Comunicación aumentativa y alternativa mediante tecnologías de apoyo para personas con discapacidad. TED: Tecné, Episteme y Didaxis, 13(2003), 1-17.

Sánchez-Montoya, R. (1998). Ordenador y discapacidad: Las entradas y los sistemas de acceso. Comunicación $y$ pedagogía: Nuevas tecnologías y recursos didácticos, 150, 30-42.

Sotillo, M. (1993). Sistemas alternativos de comunicación. Madrid: Editorial Trotta.

Tetzchner, S.V. (1993). Desarrollo del Lenguaje Asistido. Infancia y Aprendizaje, 64, 9-28.

Tetzchner, S.V., \& Martinsen, H. (1993). Introducción a la enseñanza de signos y al uso de ayudas técnicas para la comunicación. Madrid: Visor.

Valero, C., Redondo, M., \& Palacín, A. (2012) Tendencias actuales en el uso de dispositivos móviles en educación. La educación digital magazine, 147, 1-21.

Vergara, M. (2008). La naturaleza de las representaciones sociales. Revista Latinoamericana de Ciencias Sociales, Niñez y Juventud, 6(1).

Villa, M., \& Martínez, L. (2008). Comunicación aumentativa y alternativa. Disponible en http://www. jmunozy.org/files/9/Necesidades_Educativas_Especi ficas/aula_pt/conocer_mas/diversidadmurcia/UNIDAD26. pdf 